\title{
Analysis of Dynamical Electron Backscatter Diffraction Patterns of Ferrite and Martensite Phases in Steels
}

\author{
Anita Heczel and Luke Brewer
}

University of Alabama, Tuscaloosa, Alabama, United States

Electron backscatter diffraction (EBSD) is a very powerful method for characterizing the microstructure of crystalline materials. Traditionally, the Hough transform is used to find the Kikuchi bands and then index the EBSD patterns. Separating some phases in steels such as austenite and ferrite is straight forward, but this approach has difficulty differentiating phases with only slight changes in lattice parameters or highly similar space groups. Therefore, phases such as ferrite (body-centered cubic, BCC) and martensite (body-centered tetragonal, BCT) in steels cannot be separated by Hough-indexed based EBSD due to their similar crystallographic structure. For metallurgical applications, the ability to consistently and readily distinguish between ferrite and martensite is highly valuable. A recently developed EBSD-dictionary indexing method, based upon dynamical diffraction simulations, has proven to be a powerful tool in multiphase specimens [1,2]. The dictionary indexing was successfully applied to differentiate between phases in multiphase materials where Hough-based indexing was difficult or impossible [2,3]. Furthermore, the dynamical simulation of the EBSD/Kikuchi patterns is a promising method for analyzing lattice tetragonality and identifying BCT vs BCC regions [4] and study the local tetragonality with microscopic resolution [5].

The present study examines the differentiating features in simulated EBSD patterns of ferrite and martensite. The martensite phase (BCT) differs from ferrite (BCC) in that it has a tetragonal lattice distortion caused by interstitial carbon atoms; it follows that the tetragonality depends on the carbon content. We employ full dynamical patterns simulations, implemented in the open-source EMsoft software platform [1]. In this approach, a master EBSD pattern is generated covering the entire Kikuchi sphere using the geometrical parameters of the experimental setup and the detailed crystal structure information, including space group, lattice parameters, and atomic positions/occupancy. Individual EBSD patterns can be dynamically simulated from this master pattern for any crystal orientation. EBSD patterns were dynamically simulated for the martensite phase with different site occupancy carbon content (2, 4 and 6 $\% \mathrm{C}$ ), and compared to the ferrite (small sections of the Kikuchi sphere are shown in Figures 1a and 1b).

Clear differences between the ferrite and martensite (6\% carbon content) EBSD patterns are apparent by examining the subtracted difference between the $<010\rangle$ zone axis patterns for the two phases (Figure 1c). Qualitatively, the largest differences between the two EBSD patterns are at the center of the zone axis pattern and at the position of $\langle 010\rangle$ zone axis in the lower portion of the simulated EBSD pattern. The quantitative differences can be seen by plotting line profiles through three low-index bands, (200), (002), and (101), around the $<010>$ zone axis (Figs. 1a and 1d-f). A line profile was measured at the same positions in all patterns. Clear differences in band intensity for ferrite and martensite are present in all profiles, but only specific positions in reciprocal space show sensitivity to carbon concentration in the martensite. For example, in the center of the (200) band, there is no predicted difference for martensite as a function of carbon content, but other positions, e.g. at pixel position 34 in the (200 band), do show some possibility for differentiation between martensite with different carbon content.

In this talk, we will compare these simulations to experimentally measured EBSD patterns of ferrite and martensite. The simulations suggest that indexing algorithms that focus upon particular portions of reciprocal space may be more effective in differentiating between these two phases while in orientation 
mapping mode. Even more careful analysis of high quality, single patterns might be used to quantify the effect of carbon content on the lattice distortion in martensite.

Acknowledgments: This research supported by Dr. J. Wolk at the Office of Naval Research under grant ONR-N00014-18-1-2266.
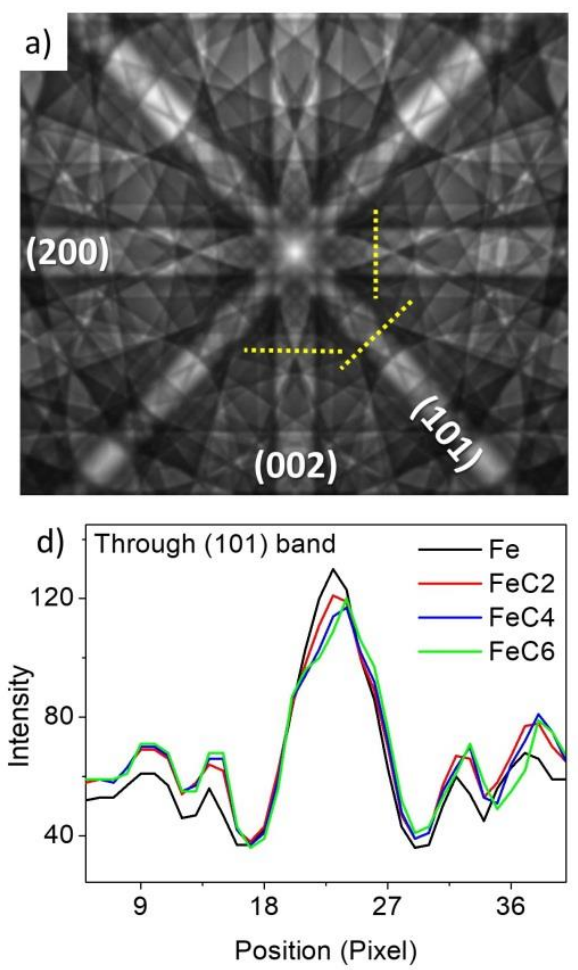
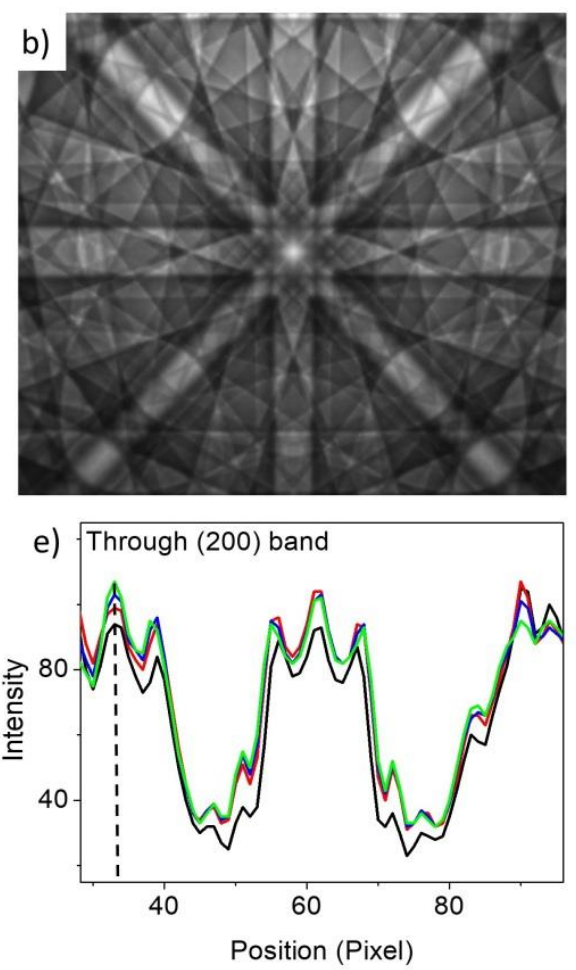
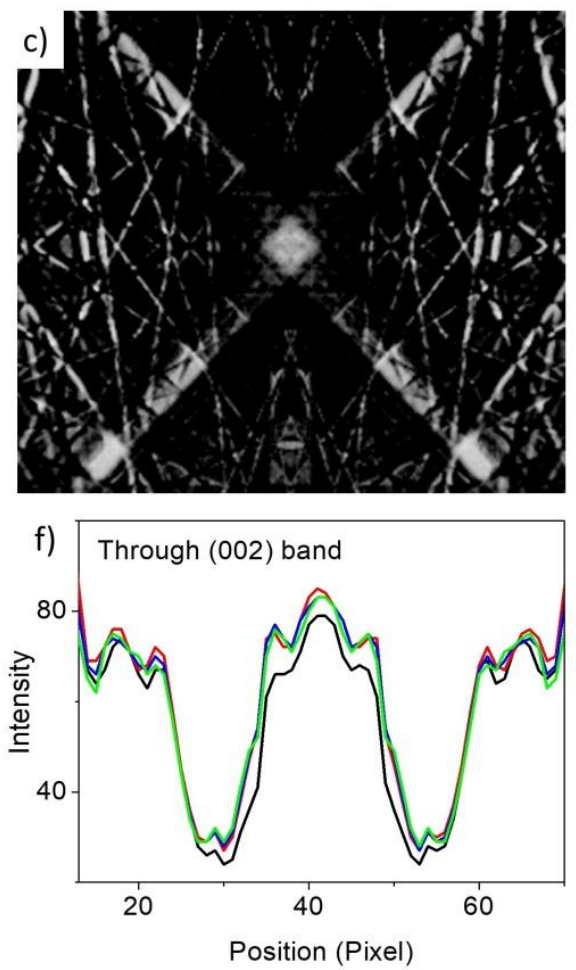

Figure 1. Figure 1. The dynamically simulated pattern by EMsoft for (a) iron ferrite and (b) martensite with $6 \%$ carbon and (c) shows the pattern difference between ferrite and martensite obtained by subtracting (a) from (b). The band profiles were measured along the yellow dashed lines through the (200) (d), (002) (e), and (101) (f) bands, respectively.

\section{References}

[1] P.G. Callahan and M. De Graef, Microscopy and MicroAnalysis, 19 (2013) p. 1255

[2] F. Ram and M. De Graef, Acta Materialia 144 (2018) p. 352

[3] L. Morales-Rivas et $\quad$ al., Scripta $\quad$ Materialia $194 \quad 113644$ (2021) https://www.sciencedirect.com (accessed November 30, 2020)

[4] D. Adams et al., J Microscopy 12980 (2020) https://onlinelibrary.wiley.com (accessed November 23, 2020)

[5] A. Winkelmann et al., Phys. Rev. Materials 2123803 (2018) https://journals.aps.org (accessed December 28, 2018) 